\title{
Percepciones y significados que tienen los trabajadores de la plaza de mercado de Ayapel (Córdoba, Colombia) sobre la Ciénaga de Ayapel.
} Meanings and perceptions of Ayapel marketplace workers about Ayapel Swamp (Córdoba, Colombia).

Martha Cenelia Ortiz Tapias ${ }^{1}$, Trabajadora Social; Lina Yoelys Jaller Vides², Trabajadora Social; Luz Helena Barrera Pérez $^{3 \star}$, Trabajadora Social, Magíster; Claudia Patricia Isaza Cruz ${ }^{4}$ Trabajadora Social, Magíster.

${ }^{1}$ Egresada de la Universidad de Antioquia, 2008. Investigadora del trabajo: “Ciénaga de Ayapel, Córdoba: Tesoro, Esperanza y Vida. 2007-2008”. Teléfono y correo electrónico: 8391155 - 3117290522 , Correo electrónico: marthacenelia@gmail.com;

${ }^{2}$ Egresada de la Universidad de Antioquia, 2008. Investigadora del trabajo: "Ciénaga de Ayapel, Córdoba: Tesoro, Esperanza y Vida. 2007-2008”. Trabajadora Social en la Comisaria de Familia en Niñez y Juventud. En 2009 trabajó con la Fundación Aso San Jorge. Teléfono y correo electrónico: 054-770-54-30 - 313-528-15-57 Lirica26@hotmail. com;

${ }^{3}$ Magíster en Salud Pública y docente en la Facultad Nacional de Salud Pública de la Universidad de Antioquia;

${ }^{4}$ Magíster en Salud Pública de la Universidad de Antioquia y Funcionaria del Municipio de Medellín adscrita a la Secretaria del Medio ambiente.

Aceptado: enero 28 de 2013.

\section{Resumen.}

Esta investigación cualitativa, de Enfoque etnográfico, pretendió comprender las percepciones y significados que los trabajadores de la plaza de mercado tienen acerca de la Ciénaga de Ayapel. Los datos se recolectaron mediante entrevistas semi estructuradas, revisión de fuentes secundarias y la observación. Los hallazgos más relevantes dan cuenta de sus problemáticas socioculturales, ambientales y económicas, en contraste con lo que perciben y lo que significan para ellos la Ciénaga y su relación con su oficio y el contexto. Los resultados buscan que ellos los aprovechen y se reconozcan así mismos, sus prácticas cotidianas y el medio que les rodea, de manera que puedan propender por un desarrollo sostenible, minimizando los impactos que genera su actividad creando alternativas para una mejor relación con el entorno.

Palabras clave: contaminación ambiental, medio ambiente, percepciones, representaciones sociales, significados.

Para citar este artículo: Ortiz MC, Jaller LY, Barrera LH, Isaza CP. Percepciones y significados que tienen los trabajadores de la plaza de mercado de Ayapel (Córdoba, Colombia) sobre la Ciénaga de Ayapel. Rev Humanismo y Sociedad, 2013; Volumen 1: 22-36.

*Autor para correspondencia: Luz Helena Barrera. Docente Departamento de Ciencias Básicas. Coordinadora Grupo de Desarrollo Académico Salud y Sociedad. Facultad Nacional de Salud Pública, Universidad de Antioquia, Medellín, Colombia. Oficina 205.

Revista. Humanismo.Soc. 2013; 25: 22-36. 


\begin{abstract}
.
This qualitative study used an ethnographic approach to understand the meanings and perceptions marketplace workers have about the Ayapel Swamp. Data were collected through semi-structured interviews, review of secondary sources, and observation. The most relevant findings show the sociocultural, environmental, and economic issues of the people, as opposed to their perception and significance of the Swamp, and also its relationship with their work and context. It is intended for this people to use and take advantage of the study results and recognize themselves, their daily activities, and their environment, so they can guide their own development under a sustainable approach, minimizing the impacts generated by their activity, and creating alternatives for a better relationship with the environment .
\end{abstract}

Key words: environment, environmental pollution, meanings, perceptions, social representations.

\section{Introducción.}

Muchas son las problemáticas que aquejan al mundo y constituyen una amenaza para la vida del ser humano, una de ellas y la más preocupante, es la contaminación ambiental que con el transcurrir de los años, ha venido ejerciendo gran impacto sobre el medio ambiente. Problemas como el orificio en la capa de ozono, contaminación del agua, aire, suelo, etc. son a lo largo y ancho del planeta, problemáticas que atentan con toda forma de vida existente, terminando así, con lo más valioso que posee el ser humano: su medio ambiente.

En Colombia la contaminación ambiental se ha convertido en una de las problemáticas claves a trabajar desde políticas y planes desde todos los ámbitos, como vía para lograr minimizar éste grave problema, para direccionar y ubicar de manera específica lo que se quiere trabajar en el plano ambiental.

La situación ambiental actual del municipio de Ayapel Córdoba, no es diferente a la de muchos otros lugares del país, pues a pesar de poseer una de las

ciénagas más importantes a nivel nacional, no existe una verdadera conciencia ambiental por parte de sus habitantes, permitiendo conservar de manera adecuada tan importante riqueza.

En este proyecto de investigación cualitativa, partió, para conocer esta realidad social, era necesario describir las percepciones y significados que tenían los trabajadores de la Plaza de Mercado sobre la Ciénaga de Ayapel, esto permitió recolectar datos importantes para la investigación, presentada de manera tal, que da cuenta del contexto dentro del cual se realizó el estudio etnográfico, seguido de una breve descripción del origen de la Plaza de Mercado y la percepción del riesgo ambiental por contaminantes, por lo que se hace necesario citar las Representaciones sociales para guiar la descripción de las percepciones en las que los trabajadores evocan el pasado y lo relacionan con todo lo que les sucede.

También se describen las vivencias del gremio investigado en relación con el problema de la contaminación y lo que significa la ciénaga para ellos. Finalizando con algunas posibles soluciones planteadas por los trabajadores de la plaza, desde lo que cada uno percibe y considera más importante, seguidas de conclusiones y recomendaciones consideradas importantes desde el Trabajo Social; y las cuales, deben ser tenidas en cuenta para continuar con otras investigaciones o para un futuro proyecto de intervención con el gremio investigado, entre otros.

La investigación fue llevada a cabo con los trabajadores de la plaza del mercado, ubicada a orillas de la ciénaga, pues es en esta zona donde mayor contaminación se ha dado debido a la actividad pesquera, a la venta del productos y al manejo inadecuado de los residuos generadas por esta actividad, que sumado al "ineficiente sistema de alcantarillado" (COLOMBIA, AYAPEL-CÓRDOBA) del municipio van deteriorando la ciénaga.

La Ciénaga de Ayapel, Córdoba, es el principal sustento económico y alimenticio para las familias más vulnerables del municipio, y a pesar de esto ellos no valoran la importancia que esta encierra y mucho menos propenden por un desarrollo sostenible, permitiendo 
un equilibrio en el proceso de su explotación. Sumado a esto, "la ciénaga abastece a la población de agua para el consumo humano, la cual no cuenta con el tratamiento adecuado, lo que en algunas ocasiones trae consigo problemas asociados con la salud como: Infección respiratoria aguda (IRA), Enfermedad Diarreica Aguda (EDA), Dengue y Malaria" (COLOMBIA, AYAPELCÓRDOBA) 4 .

Se han realizado investigaciones alrededor de la contaminación de la ciénaga por parte de varias universidades y grupos de investigación, pero lo novedoso de esta investigación, es que nunca se han descrito las relaciones hombre-naturaleza desde las percepciones y significados que tienen los trabajadores de la plaza hacia la ciénaga.

Desde el Trabajo Social, se aportan elementos importantes para que, con la participación de los actores involucrados en esta situación, es decir, los trabajadores de la plaza de mercado, se concienticen y aprovechen la investigación para reconocerse a sí mismos y al medio que les rodea, de manera que puedan propender por lograr hacer conciencia de las relaciones que están estableciendo con la ciénaga, de sus prácticas y de la transformación de las mismas.

Por ello, la investigación estuvo enfocada a conocer ¿Cuáles son las percepciones y significados que tienen los trabajadores de la Plaza del Mercado del Municipio de Ayapel Córdoba, sobre la Ciénaga de Ayapel? y, ahondar en respuestas a interrogantes generados a partir de: ¿Qué significa la ciénaga para ellos?, ¿Cuáles son sus percepciones?, ¿Cuáles son las relaciones de grupo que se dan entre ellos?; logrando de esta manera, describir la problemática desde otro ángulo más significativo y revelador, en el momento de plantear alternativas de solución acertadas.

La contaminación ambiental es una problemática que toca cada rincón del planeta y aunque parezca imposible, esto se puede evitar. Todo inicia desde el ser mismo y es a partir de allí donde brillan las percepciones y los significados haciendo de la vida un disfrute de todo lo que la rodea, deleitándose del aire, la tierra y el agua. Es por ello que el ser humano debe considerar seriamente que su relación con el medio ambiente debe ser modificada, pues quien más se está perjudicando es él mismo. Su condición de ser pensante, debe hacerlo reaccionar para buscar y lograr la forma de vivir en armonía con la naturaleza. Los recursos naturales deben ser utilizados pensando en las generaciones futuras a quienes no se les puede entregar un ambiente absolutamente deteriorado, por el contrario, considerar que los aspectos físicos, los seres vivos y los factores socio- culturales conforman un solo mundo.

Este tipo de investigaciones son de gran importancia tanto para los trabajadores de la plaza de mercado, la ciénaga misma, como para el resto de la población Ayapelense. Permite estudiar, analizar, comprender problemáticas y relaciones dadas entre los sujetos y su entorno, de lo que el ser humano haga por él, depende su conservación, su durabilidad.

\section{Metodología.}

El estudio ha sido realizado a través de una investigación cualitativa, lo que promovió el análisis de realidades sociales complejas y dinámicas desde otra mirada, más flexible, más integradora, una mirada que posibilitó la descripción de actitudes y sentimientos dados en determinados contextos. De acuerdo con Bonilla y Rodríguez (1995), la principal característica de la investigación cualitativa es su interés en captar la realidad social a través de los ojos de la gente que está siendo estudiada, es decir, de la percepción que tiene el sujeto de su propio contexto, esto indica que la investigación cualitativa, busca interpretar y comprender la estructura, dinámica y compleja del sistema representacional, así como llegar a la plena comprensión de los significados, las expresiones, las interpretaciones y explicaciones de las Representaciones Sociales, dando cumplimiento a los requisitos de credibilidad, contextualización, recurrencia, saturación y posibilidad de transferencia, propios de este modelo.

Dentro la investigación cualitativa se encuentra el enfoque etnográfico, surgido como una metodología que propicia la comprensión de una problemática a través de la reflexión que un grupo de personas hace de sus creencias, sentimientos y prácticas cotidianas y el significado de cada una de ellas en un contexto determinado.

Como técnicas de recolección de la información se utilizaron la entrevista semiestructurada, la observación y el diario de campo. En cuanto a la entrevista tuvo la 
particularidad, en algunos casos, de seguir el modelo de una conversación entre iguales y no de un intercambio formal de preguntas y respuestas y para ello se utilizó un guión de entrevista, facilitando la exploración de las categorías iniciales, consideradas importantes para el logro de los objetivos de la investigación. La guía fue flexible con el fin de recoger el flujo de información particular de los participantes.

Para la selección de la muestra se tuvo en cuenta como criterio de inclusión aquellas personas que trabajaran o tuvieran su actividad comercial en la Plaza de Mercado de Ayapel-Córdoba. Se escogieron diez personas adultas, cuya actividad fuera ser pescadores, comerciantes o vendedores y vendedoras. Todos estos identificados como personajes primordiales dentro del proyecto, por su experiencia, disponibilidad, antigüedad y relación con la Plaza y la ciénaga facilitando el abordaje de los temas a describir. Las personas a entrevistar fueron ubicadas a través de informantes claves, quienes hicieron el puente entre las investigadoras y los entrevistados.

Las entrevistas se transcribieron y se organizaron los datos en documentos de Word y de forma manual. En un documento impreso se realizó la codificación y la categorización para facilitar el análisis de los datos obtenidos.

Este método permitió interpretar la información registrada, identificando temáticas centrales $y$ secundarias presentes en el proyecto, integrándolos en una estructura descriptiva general, incluyendo la particularidad de los datos de los participantes. Es importante destacar los asuntos éticos en la etnografía, relacionados con las características de las metodologías cualitativas, las cuales incluyen un compromiso de largo tiempo y muy cercano, entrevistas y observaciones, esto es, según el Código de Ética de la Asociación Sociológica Norteamericana (Boyle, 2003), el cual hace referencia a la protección de la confidencialidad y el anonimato con sentimiento informado cuando los riesgos de la investigación son mayores que los de la vida diaria. En relación a ello, se informó a los trabajadores de la plaza del mercado, de la intencionalidad de la investigación; se respetó la cultura con la que se interactuaba, su cotidianidad y como muestra de transparencia y validez de la información sistematizada, se programará una reunión en la plaza del mercado al finalizar la investigación, para hacer la devolución a los trabajadores, de los resultados de la misma, aportando las estrategias que se pueden implementar en futuras investigaciones o intervenciones, para la concienciación de los trabajadores en cuanto al manejo de los residuos y cuidados de la Ciénaga de Ayapel, Córdoba.

\section{Resultados.}

Entre el recuerdo, el aprecio y lo que se percibe...

El Mercado Público, está localizado en el Barrio Centro del Municipio de Ayapel y es el punto de intercambio comercial más importante del municipio. Por su localización a orillas de la ciénaga en medio de un sector residencial, impacta fuertemente el medio ambiente, el urbanismo inmediato, por el gran volumen de desechos que produce y que son vertidos directamente a los cuerpos de agua, y el deterioro formal del entorno por cambios abruptos y desordenados de los usos del suelo, pasando de lo residencial a una mixtura con lo comercial. (COLOMBIA, AYAPEL-CÓRDOBA).

La Plaza de Mercado es reconocida legalmente en el año de 1995, cuando por primera vez se logran organizar los trabajadores en sus respectivos espacios, pues todos se encontraban ubicados de manera desorganizada, tal y como lo manifiesta uno de los entrevistados: “...Cuando llegué a la Plaza como tal no existía, o sea las paredes, el techo, piso, nada de esto estaba; trabajábamos bajo una rancha que cada quien se hizo y no estábamos organizados como ahora, todos estábamos revueltos: los pescadores, las vendedoras de comida, los comerciantes y demás trabajadores de la Plaza”. (E3: P11).

Luego, la Administración Municipal decide construir la plaza y organizan, a los trabajadores, de acuerdo a su actividad económica: los vendedores de comida y los comerciantes a un lado y los pescadores en otro y, para estos últimos, la ubicación fue en el mismo lugar donde estaban situados antes de la construcción, cerca de la ciénaga.

Sin embargo, los trabajadores de la Plaza comienzan a construir su realidad desde mucho antes, es decir, desde el año 1982, a través de la interacción sostenida con el medio; por lo que la ubicación de la plaza de Mercado generó cambios y facilidades para la población ayapelense, permitió mejorar la situación económica de muchas familias. 
Las trabajadores de la Plaza de Mercado se caracterizan por ser personas de escasos recursos económicos, algunas son madres solteras, trabajadores independientes, todos con una familia que sostener. Llevan entre 17 y 25 años en esta labor y se situaron en la plaza para vender lo que cosechan, pescan, o cocinan, o para re vender lo que compran. No cuentan con la posibilidad de acceder a otro tipo de empleo por razones como: bajo nivel educativo, desempleo, o porque simplemente les gusta, entre otras, aunque no represente las mejores condiciones de vida comercial.

También, se caracterizan por su solidaridad, reconocen las necesidades del otro y se ayudan mutuamente en lo que esté a su alcance. En muchas ocasiones se les dificulta ponerse de acuerdo como grupo para realizar alguna actividad, aunque reconocen la importancia de la presencia de un líder que los represente ante los entes gubernamentales, especialmente ante la Alcaldía Municipal, pues el no tenerlo es una razón para que no los tengan en cuenta en sus proyectos.

Para Deschamps y colaboradores: "Los individuos piensan sus grupos a partir de un conjunto fluido de atributos, el prototipo, una imagen que maximiza la diferencia entre grupos de pertenencia y de nopertenencia, la cual permite, al mismo tiempo, reforzar la idea de una semejanza existente en el interior de los grupos" (Deschamps, Morales, Paez, \& Worchel, 1999). Desde las Representaciones Sociales, para los trabajadores de la Plaza de Mercado de AyapelCórdoba, es notorio la importancia de reconocer a sus compañeros como miembros de un grupo en función de un 'aire de familia' dada la convivencia diaria, a la semejanza y a las cosas en común que existe entre ellos, tales como: la lucha por subsistir el día a día, las actividades económicas, opiniones, percepciones, $\mathrm{y}$ por supuesto, la relación que cada persona tiene con la ciénaga, ésta constituye el factor más común existente entre ellos y toda la población ayapelense.

Pese a las diferencias, ellos se reconocen como gremio, que necesitan mejorar sus relaciones de grupo, pues es necesario y "es de naturaleza misma de la categorización de ser un proceso cognitivo de reagrupamiento que transforma diferencias en semejanzas y viceversa" (Oakes PS, Haslam A y Turner JC, 1999).

Es necesario agregar que los Trabajadores de la Plaza de Mercado no sólo tienen una historia común, rasgos y formas de actuar conocidas entre ellos, sino también se construye una historia de convivencia, de peleas o de alianzas, un conocimiento mutuo, afectos culturales entre ellos, ya sean positivos o negativos, pues esto también moviliza la energía de los participantes o miembros del grupo.

Es la memoria compartida de lo que ha sido vivido en común, como individuos y como miembros de una colectividad, reconstruida a instancias de interacciones sociales y condicionadas por los marcos sociohistóricos en los que se produce. La memoria colectiva se construye como resultado de las interacciones múltiples de los miembros de un grupo, de manera tal que lo que cada miembro del grupo recuerda es producto de esa interacción [que se da al interior] de marcos sociohistóricos [...] conformados, fundamentalmente, por el intrincado tejido institucional bajo cuya normativa interaccionan los miembros del grupo" (Agudo Guevara, 2003).

La memoria compartida es también constitutiva de las experiencias del presente, la manera de enfrentarlas y de definir el futuro. Se debe tener en cuenta que no siempre la historia de un grupo o comunidad está documentada por escrito. Cuando no existen escritos históricos al respecto, tal como sucede en este caso, la memoria social permite reconstruir ideas, cambios o hechos a través de la evocación de los recuerdos en el presente, pero, hay elementos nuevos e inesperados irrumpiendo en el presente, que rompen la continuidad de la vida cotidiana, levantan una barrera entre presente $\mathrm{y}$ futuro: plantean un conflicto por resolver.

George Herbert Mead y Moscovici coinciden en acordar una importancia fundamental a los elementos nuevos, extraños que penetran en la vida cotidiana, a los cuales denomina "eventos emergentes de experiencia". La novedad es, para Mead "el cimiento de la conciencia, la inteligencia y la libertad de acción; es el fundamento básico de la experiencia humana” (Mead, 1938), “Toda acción humana es acción en el tiempo y sin la ruptura de la continuidad del presente que introducen los eventos emergentes no podría haber ningún tipo de experiencia, sólo habría un mero pasaje de eventos" (Banch , 2006). Es por ello, que dentro del contexto de esta investigación la experiencia comienza con lo problemático; con la ruptura de la continuidad. Si no hubiese rupturas de la continuidad, no se tendría conciencia de ella. 
Es cierto que no hay un pasado irrevocable, porque el pasado es reinterpretado para dar sentido a los eventos del presente. Y también es cierto, que en el pasado algo sucedió, pero la respuesta a la pregunta sobre qué sucedió es una respuesta sujeta a múltiples reinterpretaciones; la conciencia histórica no sólo permite comprender el cambio sino también impulsarlo de tal forma que el individuo o grupo, históricamente mentalizado, pueda contribuir al desarrollo de nuevas estructuras dentro del proceso del tiempo; y he ahí la importancia de conocer o indagar sobre el pasado que relaciona a los protagonistas de esta investigación.

La importancia de la evocación de los recuerdos de los Trabajadores de la Plaza de Mercado, ahora en el presente, permite mostrar los esfuerzos, el origen de sus percepciones, de sus significados, denotando que "El recurso histórico del pasado se asienta sobre las situaciones nuevas emergentes en el presente y que sugieren un futuro. El pensamiento humano, incluyendo la conciencia histórica, es una confrontación con la novedad cuya meta es pasar de un presente a un futuro no problemático. (...) La meta de la historia es el establecimiento de una sociedad en la cual todos y cada uno van a reconocer los intereses de todos los demás, una sociedad cuya regla de oro es una regla de conducta, esto es, una sociedad en la que cada quien hace de los intereses de los demás su propio interés." (Mead, 1938).

\section{"Si queda será un moridero..." El riesgo ambiental percibido.}

Para hablar de las percepciones, también es preciso hablar de las representaciones sociales, siendo ésta "una manera de interpretar y de pensar la realidad cotidiana, una forma de conocimiento social. Y correlativamente, la actividad mental desplegada por individuos para fijar su posición en relación con situaciones, acontecimientos, objetos y comunicaciones que les conciernen" (Jodelet D. , 1988).

La Ciénaga de Ayapel es el humedal más importante para los ayapelenses, sus vientos son fuertes, sobre todo en la noche, es de una belleza paisajística incuestionable. Toda vez que se le pregunta a un ayapelense cómo era la ciénaga antes, denota una mirada y una sonrisa nostálgica, evocando el pasado y lanzando un suspiro responde con tristeza “...antes nos íbamos a bañar con la familia allá, pero ahora no se puede hay mucha basura en ella" (E2:P7). “imagínese teníamos agua cristalina que se podía tomar con toda tranquilidad, lo mismo que bañarse en ella sin pensar que después nos fuera a dar rasquiña, los manglares, los peces..." (E6:P33).

En cada cultura las representaciones pertenecen a la comunidad, y la comunidad misma es co-construida por la gente en sus prácticas sociales y conversaciones cotidianas. Las representaciones "son elaboraciones de grupos sociales que sirven para mantener la estabilidad de su mundo de vida particular [...], están atadas a contextos sociales, esto es, atadas a grupos y sus mundos de vida y, a situaciones y eventos que ocurren en esos mundos de vida y que requieren formas específicas de pensar, hablar y actuar" (Wagner, Duveen, Verna , \& Thelme, 2000).

Los Trabajadores de la Plaza de Mercado perciben muchas situaciones, sobre todo de riesgo a perder algo significativo en sus vidas, hay que tener presente que "La mayoría de las percepciones, son el resultado de la capacidad del individuo de sintetizar las experiencias del pasado y las señales sensoriales presentes" (Ferdinand von Hermholtz, 1989), y dentro del contexto de esta investigación, la percepción del riesgo, cualquiera que sea, es el resultado de la interacción de procesos cognitivos, emocionales, interpretativos y evaluativos que hacen que el individuo se haga una idea del entorno donde vive y lo contextualice, haciendo conciencia del mismo.

Tal es su conciencia del riesgo que sus actividades cotidianas traen al entorno que uno de ellos dice en su entrevista:

"Si la ciénaga sigue así, desaparece seño, ella de por sí ya está bien contaminada y si se sigue, imagínese, no queda nada y si queda será un moridero, toda la belleza sería historia”. (E6:P34)

No obstante, también existen algunas personas que tienden a ser resistentes a la idea de que se encuentra en riesgo frente a determinado peligro, este optimismo irreal se sustenta en la información disponible y en un razonamiento que induce a pensar que el peligro no es una amenaza verdadera, aunque afecte a personas conocidas.

Se ha argüido que la respuesta al peligro está mediada por influencias sociales trasmitidas por amigos, la 
familia, los compañeros de trabajo y los oficiales públicos respetables. En muchos casos, la percepción del riesgo se puede formar después de un hecho racional ejecutado por el propio individuo" (Urbina \& Fregoso, 1991).

Vale la pena mencionar a Puy (1995), quien afirma que la comprensión de la percepción del riesgo desde las ciencias sociales, supone el estudio de las creencias, actitudes, juicios y sentimientos, así como los valores $\mathrm{y}$ disposiciones sociales y culturales que las personas adoptan frente a las situaciones poniendo en peligro el lugar que aman o representa algo; en este caso, la contaminación es un riesgo para la conservación de la ciénaga, de sus aguas, del aire y por ende, de la salud.

Cuando se les pregunta cómo se imaginan al municipio sin la Ciénaga, ellos responden "Un cementerio más de lo que es, lo único que le da vida a Ayapel es la ciénaga” (E3:P16). Pese a que son conscientes del daño a la ciénaga y de las posibles soluciones, no las ponen en práctica.

Sumado a lo anterior, la mayoría de los Trabajadores de la Plaza de Mercado se muestran como personas sensibles ante la ocurrencia de una situación medio ambiental, como la que se ha venido presentando desde años atrás en la Plaza con relación a la ciénaga de Ayapel. En este sentido, fue común encontrar frases sentimentales que evocan recuerdos en casi todos los entrevistados "...Para mí es un tesoro que no lo sabemos valorar, pero es un tesoro, porque si no fuera por ella mucha gente moriría de hambre y muchos no están empleados y viven de ella, del pescado" (E5:P25).

Agua que no has de beber...no la ensucies.

"La ciénaga de Ayapel es de una belleza paisajística incuestionable. Pero se está muriendo lentamente debido al proceso acelerado y permanente de contaminación en que se encuentra sometida", afirmó el ex presidente del Concejo de Ayapel, Fernando Márquez Martínez. Esa misma percepción tienen otros: "Tenemos una hermosa laguna, aunque ahora fuera mucho más hermosa por la variedad de peces que habian, manglares, pájaros, pero ahora sólo quedan unos cuantos, todo se ha ido perdiendo poco a poco" (E6:P32).

En la Plaza de Mercado de Ayapel, algunas personas han tenido una percepción similar de los peligros ambientales que enfrenta la comunidad porque han sido más observadores de su entorno y comprenden lo que en él ocurre; ese espacio donde permanecen mucho tiempo, se convierte en su segundo hogar y es precisamente por ese tiempo, que su dependencia y su experiencia personal tienen un papel fundamental a la hora de componer la imagen del medio.

Cuando los trabajadores no están en la Plaza de Mercado, aprovechan para participar en asuntos a los que no les han dedicado mucho tiempo, como el hogar, a Dios y otros visitan la ciénaga sin olvidar lo que representa para ellos.

Todo esto, llevó, a los trabajadores de la Plaza de Mercado, a crear una imagen de ésta y de la ciénaga, común a todos ellos, sin dejar de lado el componente afectivo y simbólico, al constatar la importancia de las vivencias y el valor emocional en el sujeto a la hora de la representación, convirtiendo a éstos espacios en 'lugares' cargados de significación "La ciénaga es el reflejo de amor y amistad, es muy bonito, el que viene llega a ver la ciénaga, y además, alimenta a muchas familias, es una fuente de trabajo. Su nombre significa "Acuario de amor y amistad" (E5:P26), "La ciénaga significa mucho para mí, es un espacio de recreación y un panorama cultural, o sea, como algo histórico" (E4:P21).

La Ciénaga también representa una fuente ingresos y para muchos es la única forma de ingresos económicos para el sostenimiento del hogar o de sí mismo. En el municipio de Ayapel se hace necesario mantener y ampliar las actividades económicas por el significado social que ellas tienen en la generación de riquezas; pero al mismo tiempo se debe tomar conciencia sobre la contaminación ambiental que éstas causan, para buscar soluciones y mantener el equilibrio ecológico y ambiental.

Desde las representaciones sociales se privilegia igualmente el análisis de procesos del pensamiento social, los cuales constituyen "modalidades de pensamiento práctico, orientado hacia la comunicación, la comprensión y manejo del ambiente social, material e ideal" (Jodelet D. , 1988). En la opinión de Banch, Moscovici comparte con Meadla idea de quelas acciones construyen la realidad, cosa evidente cuando expresa que desde las representaciones sociales "El sujeto y el objeto no son considerados como funcionalmente 
separados. Un objeto se localiza en un contexto de actividad en la medida en que es lo que es porque es en parte considerado por la persona o por el grupo como una extensión de su conducta. No reconocer el poder de nuestra capacidad para representar o crear objetos y eventos es como creer que no hay conexión entre nuestro reservorio de imágenes y nuestra capacidad para la imaginación" (Moscovici, 1973).

El único problema que tenemos es el de las basuras... Lo incómodo.

Las personas entrevistadas en la Plaza de Mercado platican acerca de las diversas situaciones vividas diariamente, dando cuenta de situaciones incómodas que los marca y los entristece, tales como: las consecuencias de la contaminación, la falta de un líder, las relaciones de gremio, la falta de compromisos y el deficiente servicio de recolección de basuras.

La contaminación ambiental es una problemática que día a día toma mayor fuerza e importancia debido al crecimiento acelerado de la industria, generadora de gran cantidad de desechos contaminantes que van directo a afectar al Medio Ambiente, entendiendo este "como un complejo sistema de interrelaciones que lo componen muy activo entre el espacio físico $y$ el sociocultural" (MINISTERIO DEL MEDIO AMBINETE).

En el municipio de Ayapel, esto se evidencia en la manera en que todos los desechos originados por la población, especialmente de la Plaza de Mercado, culminan su recorrido en la Ciénaga, aumentando de este modo, el grado de contaminación, la disminución de las especies nativas de la laguna y consecuentemente la desaparición de esta hermosa fuente hídrica.

Todo esto, muestra los efectos ecológicos de la acción humana sobre el hombre y su dimensión sociocultural, entendidos como las derivaciones que el gran desarrollo tecnológico e industrial ha excedido y sobrepasado la capacidad de la naturaleza para restablecer el equilibrio natural alterado en el que el hombre se ha visto comprometido.

Como ya se escribió, el mayor problema de las comunidades es la basura, por consecuencia del consumo en exceso. Sin dejar de lado que el servicio de recolección de basura se torna insuficiente y la cantidad de basura adquiere otra dimensión, aún más crítica, que perturba el ecosistema. Lo anterior junto con el ineficiente manejo que se hace con dichos residuos (quemas a cielo abierto, disposición en tiraderos o vertederos ineficientes) provoca problemas tales como la contaminación, que resume "problemas de salud y daño al ambiente, además de provocar conflictos sociales y políticos" (Sánchez Homero, 1997). Esto es percibido por los trabajadores de la plaza, manifiestándolo así: “...No es nada agradable trabajar con toda esta basura encima y el olor es desagradable, tanto para nosotros los que trabajamos aquí, como para los vecinos, los clientes y peor aún, los turistas cuando nos visitan, esto da es pena" (E2:P10).

En efecto, la Plaza de Mercado y la Ciénaga de Ayapel han sido la fuente de sostenimiento para muchas familias ayapelenses, la relación entre ambas es necesaria para garantizar el sostenimiento, lo que no han podido garantizar es el bienestar de ambos espacios para que se conserven, para que la comunidad pueda disfrutarlos por mucho más tiempo.

Alrededor de este sitio existen establecimientos de comercio, tales como: restaurantes, cantinas, almacenes de ropa, kioscos, mesas de vender pescado, cuyos propietarios, junto a los trabajadores de la Plaza, vienen siendo los únicos que conocen la magnitud de lo que sucede, los dramas que les ha tocado vivir, su cotidianidad, sus sentimientos trastocados con sus actitudes y experiencias...el tiempo pasa, pero, las consecuencias no se hacen esperar.

Pese a que algunos manifiestan ser conscientes de la contaminación que generan, y luchan día a día manejando adecuadamente los desechos, se les presenta una situación difícil de solucionar, sobre todo, porque no existe el trabajo en equipo y se trata de las personas que viven en otros barrios y que en las horas de la noche, al parecer no tienen los mismos pensamientos reflexivos respecto al daño que se están ocasionando a sí mismos y arrojan basuras al lugar que si bien cuenta con una persona encargada de vigilar la Plaza de Mercado es difícil para ella controlar la situación.

La pregunta es, ¿Por qué la gente arroja las basuras a la ciénaga si existe un carro recolector encargado de llevarse estos residuos no aprovechables?. Pues bien, precisamente este es otro de los puntos negativos que existen en el municipio, los entrevistados manifiestan 
inconformidad por el servicio poco frecuente, lo que lleva a que los recipientes se llenen rápido y muchas veces los desechos se ven arrojados en la ciénaga o regados por todas partes.

En relación a lo anterior, en cuanto a la recolección, manejo y disposición final de basuras provenientes de todo el municipio de Ayapel, es realizado por una empresa particular privada, quien recoge las basuras y las deposita en el relleno sanitario del municipio de Caucasia, Antioquia, pues el municipio de Ayapel no cuenta en la actualidad con un relleno sanitario que cumpla las normas técnicas mínimas (COLOMBIA, AYAPEL-CÓRDOBA).

En el municipio es común la quema de basuras en los patios, la disposición de residuos sólidos, animales muertos y cascarilla de arroz a las ciénagas y caños, con la consecuente contaminación visual, malos olores, proliferación de roedores, insectos, aves de carroña y otras especies de animales típicos de los basureros que son propagadores de enfermedades.

En el caso específico de la cascarilla de arroz se está tratando con grandes volúmenes de materia orgánica que al ingresar a la ciénaga sufren procesos de oxidación consumiendo el oxígeno presente en las aguas y por lo tanto desequilibrando el hábitat de las especies presentes en ese medio.

Pese a los problemas por los que han pasado los Trabajadores de la Plaza de Mercado, las relaciones de gremio no han sido muy fuertes, se les dificultan reunirse y plantear soluciones para contrarrestar el problema de las basuras y, cuando logran hacerlo, los acuerdos o compromisos son muy débiles, carecen de durabilidad.

Otra situación al respecto se da porque la Administración Municipal no les presta mucha atención cuando piden ayuda para la solución al problema de recolección de las basuras. Manifiestan, además, que no se han dado cuenta de si hay algún proceso social en funcionamiento, no les han dado capacitaciones ni programas de tipo ambiental, sobre el manejo de los residuos, los desechos, entre otros, a excepción de algunas recomendaciones con débiles compromisos a seguir.

El reconocimiento de los compañeros como miembros de un grupo, por la convivencia diaria entre ellos, por las cosas que tienen en común, por lo que perciben y sobre todo por la relación de cada uno con la ciénaga les ayuda a identificar problemáticas y a la vez obtener alternativas de solución acertadas.

La contaminación si se puede disminuir... Posibles soluciones.

Cada persona no es un receptor pasivo de las influencias que su medio ambiente le impone, sino que es capaz de actuar respecto a tales influencias y reconstruir sus relaciones con el mismo, sin dejar de lado que la actitud es "como una estructura activa o proceso en la mente de un individuo, responsable de cada modificación a nivel representativo y del comportamiento" (Silvana de Rosa, 2006). Aquí las representaciones sociales son como vías del conocimiento peculiar de la realidad social, que se presentan en la vida cotidiana en el curso de la comunicación interpersonal y están dirigidas a la comprensión y al control del entorno físico-social.

La cultura no es tan sólo significados y conceptos compartidos, sino la manera como cada persona educa al otro, produce bienes y servicios y diseñan políticas sociales. En palabras de Ratner: "lo que hace cultural a los fenómenos psicológicos, no es simple hecho de que son comunes para varios individuos o de que se estimulan a través de la interacción social. Más importante es el hecho de que los fenómenos psicológicos se fundamentan sobre la actividad práctica que se organiza en un sistema social particular y concreto" (Silvana de Rosa, 2006).

De acuerdo con María Banch, la relación entre prácticas y representaciones sociales es dialéctica, no es posible pensar en ellas fuera del contexto relacional, en el escenario de la vida pública. Como tampoco se puede ignorar la estrecha relación que existe entre la manera como cada persona piensa y define su mundo y la manera cómo actúa en él y sobre él. Las representaciones hacen las prácticas en la misma medida en que éstas construyen a aquellas, y esas prácticas no pueden desligarse de una estructura social definidas por roles, normas y comportamientos aceptados o proscritos.

Las Representaciones Sociales son modos de pensar e interpretar la realidad cotidiana, son formas de conocimientosocial.Elindividuoelaboraconocimientos a partir de sus experiencias y para lograrlo, hace uso de los sistemas de significación construidos previamente. 
Esos sistemas de significación se modifican mediante la construcción de nuevos conceptos.

En relación a ello, los trabajadores de la Plaza de Mercado, aunque reconocen su responsabilidad en el caso, manifiestan que no son los únicos responsables del problema de contaminación existente, pues se encuentra la misma población, la Administración Municipal, la Gobernación de Córdoba, entre otros. Y por ello las soluciones deben partir de cualquiera de los actores sociales involucrados y contar con la participación activa de todos. Es un problema de todos y la solución es igualmente de todos.

Es por esto, que los entrevistados, desde lo que cada uno considera más importante, plantean algunas posibles soluciones que deberían ser tenidas en cuenta por la población en general: "La contaminación si se puede disminuir, pero no es solución únicamente de nosotros los pescadores; si no de toda la comunidad en general. Esto se podría lograr con la colaboración de todos nosotros. Si cuidáramos lo que se tiene y depositáramos los desechos donde deberían ser; pero no es nada fácil, porque esos serían los primeros días después el carro de la basura no vuelve a pasar y se llega a lo mismo" (E2:P8).

También, proponen análisis desde el pensamiento científico en áreas Ecológicas, sociales, psicosociales, entre otras y sobre todo plantean retos como el de buscar conocimientos relacionados con la problemática $y$, proponer proyectos de intervención, llevando al mejoramiento del medio ambiente: "Primero optar los recursos que son muchos, para ayudar a mejorar la ciénaga y luego buscar los mejores científicos, los mejores grupos ecológicos y evitar talar, organizar la alcantarilla, sacándola de allí; científicos en todos los aspectos que contribuyan al mejoramiento de la ciénaga" (E8:P64).

En estas entrevistas se logra identificar la valoración que los trabajadores tienen por la ciénaga, por la Plaza de Mercado, al igual que la falta de compromiso que evita tener prácticas adecuadas de comportamiento haciendo que la contaminación deteriore la ciénaga, aun percibiendo el futuro que le depara. Y con todo esto hay que tener en cuenta que la memoria social sirve no solamente para establecer identidad de un grupo particular, sino "como un instrumento político de reconocimiento que permite introducir una relación de poder entre los grupos sociales" (Viaud, 2002) y las minorías sociales la utilizan para hacer reconocer sus puntos de vista.

\section{Conclusiones.}

Las conclusiones de esta investigación apuntan a diferentes asuntos, algunos de los cuales se describen a continuación.

En el municipio de Ayapel hay necesidad de priorizar, mantener y ampliar las actividades económicas por el significado social que ellas tienen en la generación de riquezas; pero al mismo tiempo se hay necesidad de tomar conciencia sobre la contaminación ambiental que éstas causan para buscar soluciones y mantener el equilibrio ecológico y ambiental.

La forma en que las personas enfrentan una situación de riesgo ambiental, depende mucho de las percepciones y significados que tienen de su entorno, pero también de las relaciones que como gremio tengan, esto les facilita la búsqueda de salidas colectivas a la problemática. Una comunidad organizada puede constituirse como una comunidad fuerte, permite superar más fácilmente las eventualidades y dar soluciones precisas, que si actúan de manera individual. Por su parte los trabajadores que reconocen la existencia de la problemática y han mostrado salidas con poca fuerza a la situación y a lo que la produce, reconocen que les falta organización.

Es una realidad que la contaminación de la ciénaga es una situación que ha afectado la vida de muchas personas. Entre los límites que reconocen sus habitantes, es significativo que acepten la explicación del origen del problema como producto de la desorganización gremial y por ello, perciben el riesgo ambiental al que se enfrentan día a día; es decir, lo positivo y lo negativo que sucede en la Plaza de Mercado y en la Ciénaga de Ayapel, frente a los comportamientos o actitudes asociadas a esta problemática.

Otro de los límites igualmente reconocidos, es que no han hecho distancia total y eficaz frente a la problemática, y viven su cotidianidad entre el pasado y el presente; aunque poseen percepciones de lo que puede suceder en un futuro, aún considerado que sólo el Estado puede hacer algo por ellos.

Igualmente se han realizado proyectos relacionados con el mejoramiento de la Ciénaga de Ayapel, como por 
ejemplo, el manejo de las aguas residuales, entre otros, pero no existen proyecto en relación a la contaminación por los residuos sólidos que deja la actividad pesquera y comercial de la plaza.

La conservación de la ciénaga no es asunto únicamente de los Trabajadores de la plaza, sino también de la comunidad en general, incluyendo instituciones gubernamentales y no gubernamentales; es una problemática que, necesariamente, requiere de la participación de todos para hallar una solución acertada en vía del mejoramiento de la calidad del medio ambiente y el desarrollo de la población.

Los trabajadores de la Plaza de Mercado, no dan cuenta de verdaderos procesos ambientales que se hayan llevado a cabo para combatir la contaminación. Sin embargo, la realización de esta investigación permitió evidenciar aspectos importantes a tener en cuenta a la hora de realizar procesos de mejoramiento medio ambiental, tales como: lo actitudinal, lo gremial, lo perceptivo, lo educativo, etc.

Los trabajadores de la Plaza de Mercado, han sido, en general, más perceptivos que activos gremialmente, porque han percibido todo el tiempo el riesgo ambiental al que se enfrentan y han intentado organizarse para luchar en pro de un mejor ambiente, pero, han sido infructuosos sus esfuerzos ya que su lucha ha sido aislada y no ha contado con la participación activa y sistemática de todos los actores sociales que directa o indirectamente también hacen parte de la problemática. Ellos han tenido que luchar solos y el acompañamiento sostenido a nivel institucional ha sido sólo para remodelación de la infraestructura física, lo cual no es suficiente para minimizar el impacto de su quehacer cotidiano.

Por ello es importante que, sin generar dependencias gremiales, lasinstituciones acompañen socialmente alos trabajadores, no sólo mientras se hace la remodelación de infraestructura física, sino para que continúen, en el tiempo, realizando asesorías y seguimientos puntuales a los procesos autogestionarios.

En una remodelación, los procesos sociales y los constructivos van paralelos, pero hay que comprender que los sociales son más largos y requieren de mayor acompañamiento en el tiempo. La pertinencia de dar continuidad a lo social, es un asunto estructural dentro de lo medio ambiental que ha sido un aspecto poco desarrollado y debe ser pensado con mayor detenimiento y dedicación.

Los grupos, a través de las representaciones sociales se apropian del mundo exterior y lo pueden conocer e interpretar y construyen una realidad común, que posibilita la comunicación. Los objetos percibidos, se asocian a determinadas categorías, que difieren de pueblo en pueblo y de cultura en cultura, dependiendo de la práctica social de esos pueblos.

Se puede mediante ellas, entender y explicarse la realidad de determinados fenómenos o situación problemática, por ejemplo la contaminación de la ciénaga de Ayapel. Las representaciones sociales son, entonces, construcciones mentales que tienen autonomía y son creativas. No son reflejos del mundo exterior. Son construcciones hechas a partir de una realidad. Representan el pensamiento común, ingenuo, espontáneo y son esquemas explicativos a partir de los cuales se vive la vida cotidiana. Tienen sentido y valor para un grupo determinado que las comparte

Este es un punto fundamental para orientar este tipo de investigaciones etnográficas, su cultura sus creencias, las explicaciones ofrecidas a los fenómenos de su vida cotidiana no son mejores ni peores, ni más ni menos racionales, que las explicaciones que sobre esos mismos fenómenos pueden ofrecer los más afamados conceptos científicos.

Es preciso considerar que esta investigación no solamente permitió el intercambio de información, también permitió a los participantes conocerse un poco más y asociarse con los investigadores, los llevó a reconsiderar sus propias percepciones en relación con ellos mismos, le ayudó a interiorizar su manera de pensar y de hacer.

\section{Recomendaciones.}

"La contaminación si se puede disminuir, pero no es una solución únicamente de nosotros los pescadores; si no de toda la comunidad en general" (E2:P8)

Cada vez más las ciencias sociales, y no ya sólo las exactas o naturales, debaten desde sus perspectivas, diferentes aportes para la solución de una problemática 
que como esta, que tiene la ciénaga, exige un enfoque holístico y sistémico. Esto explica que el medio ambiente no sea la excepción y permita conocer conceptos y lugares inmensurables, dentro de un contexto social determinado, en este caso, la Plaza de Mercado y la Ciénaga de Ayapel.

La teoría de las representaciones sociales en la valoración de un contexto urbano, su lectura, su análisis e interpretación, requiere de un conocimiento profundo de la relación ser humano-entorno. Pretende saber hasta qué punto las intervenciones, que se desarrollen en un espacio específico, influyen en el desarrollo de una sociedad y también, observar cómo una sociedad puede llegar a influir en el desarrollo del entorno físico y estriba en el reconocimiento del otro como productor de conocimientos, donde los sujetos sociales adquieren el estatuto de actores comprometidos, con su realidad y el cambio.

Desde el Trabajo Social, a través de proyectos de intervención, se pueden implementar estrategias de sensibilización y educación que permitan, a través de lo social, reflexionar sobre los comportamientos dados en el entorno que, a su vez, genere actitudes positivas de prevención de problemáticas que afecten el Medio Ambiente. Esto visualizará el hacer profesional desde la relación ser humano-naturaleza a partir de las percepciones y los significados que éste construye dentro de su propio contexto.

Es decir, para resolver los numerosos problemas ambientales que tienen que enfrentar actualmente los trabajadores de la Plaza de Mercado y por ende el municipio de Ayapel, se requiere un cambio de actitud hacia el ambiente, ya que es la supervivencia humana la que está en peligro, de allí la necesidad de proteger el ambiente y se recupere ejecutando estrategias bajo el diseño de programas que permitan utilizar medidas preventivas en las diferentes instituciones del municipio.

Varias iniciativas existen para reducir o resolver el problema y, aunque dependen principalmente de los gobiernos, las industrias, las personas o de la sociedad en su conjunto; lo cierto es que esta clase de actividades puede ser realizada por los Trabajadores de la Plaza del Mercado, sin esperar la ayuda de la Alcaldía Municipal y mucho menos de la empresa encargada de recoger los desechos haga presencia, es solo que se organicen y generar estrategias para el manejo adecuado de las basuras de manera colectiva.

Para alcanzar la sostenibilidad del medio ambiente es fundamental que los recursos naturales se utilicen de forma inteligente y protegiendo los ecosistemas complejos de los que depende la supervivencia. Debe tenerse en cuenta que, la sostenibilidad no podrá lograrse con los modelos actuales de consumo y uso de recursos. Los suelos se están degradando a un ritmo alarmante. Las especies vegetales y animales están desapareciendo a un ritmo sin precedentes. Se sobreexplota la pesca y otros recursos marinos. Las personas de bajos recursos económicos son los más afectados por esta situación porque, por lo general, para subsistir, dependen de la explotación de los recursos naturales que tienen a su alrededor.

La superación de estos y otros problemas ambientales promoverá una mayor atención a la situación de los trabajadores de la Plaza de Mercado y establecer un nivel de cooperación municipal sin precedentes.

Las medidas adoptadas para detener la destrucción del medio ambiente son muestra de que es posible avanzar, siempre que exista voluntad política para ello. Todo esto, teniendo en cuenta, que el desarrollo sostenible "es el que conduce al nacimiento económico, o a la elevación de la calidad de vida y al bienestar social sin agotar la base de los recursos naturales renovables en que se sustenta, ni deteriorar el medio ambiente, o el derecho de las generaciones futuras a utilizarlo para la satisfacción de sus propias necesidades" (Jiménez Herrero, 1996).

La educación adquiere una importancia sustancial, orientada a incentivar la apropiación y valoración por parte de la colectividad de las transformaciones y cambios de su entorno. En relación a esto, se hace necesario que la Alcaldía Municipal genere estrategias de educación ambiental y en las políticas de desarrollo se incorpore este tema con mayor relevancia en los espacios locales.

Es necesaria una mirada integral a los problemas sociales y ambientales que tiene el municipio y su relación directa con el deterioro de la Ciénaga. Generar procesos participativos es el punto de partida para comprender la repercusión de las acciones cotidiana y, sus efectos sobre el entorno. 
Por ello es necesario comprometer a los individuos para encontrar entre todos soluciones viables para un futuro mejor. También es importante implementar procesos sociales que generen una cultura medioambientalista, partiendo del reconocimiento a las tradiciones culturales y a la identidad de esos territorios y, utilizando a la comunicación popular para incorporar, no sólo a los trabajadores de la Plaza de Mercado, sino también a toda la comunidad la dimensión ambiental, propiciando la activa participación de sus miembros en el diseño de una sociedad sustentable, dándole la importancia y valor a este sector del municipio.

Se evidencia la necesidad de que los trabajadores de la plaza del mercado se organicen como gremio y tomen acciones puntuales, como la elección de un líder que los represente ante los espacios de debate y de toma de decisiones, y en el ejercicio de su liderazgo, proyecte estrategias que lleven al mejoramiento de la problemática de la contaminación de la ciénaga

Para ello, es fundamental que los trabajadores de la plaza reflexionen acerca del espacio en el que se encuentran, pues éste no debe ser mirado como el que les genera recursos, sino como ese espacio vivido que les permite relacionarse e interactuar con lo que tienen a su alrededor. Vale la pena aclarar que la comunicación y la información son factores de gran importancia para lograr un nuevo equilibrio entre el individuo y su entorno; su calidad y contenido tanto como su forma y función son nuevos retos a los que se debe enfrentar.

Sensibilizar a la comunidad frente a las transformaciones de su entorno, de manera vital y consecuente con su cultura, se convierte hoy en un factor importante de transformación de la actitud del ciudadano frente a su entorno y, por tanto, en instrumento educador. Se puede aumentar la sensibilidad hacia el medio ambiente mejorando la capacidad humana de cómo se percibe el entorno.

Agregado a lo anterior, se hace necesario implementar procesos de acompañamiento profesional con metodologías grupales, donde, a través de actividades retrospectivas y prospectivas, se recoja la memoria colectiva de lo que se ha hecho para que la contaminación no avance más, para reconstruir una historia, explicar por qué ha ocurrido, resaltar la identidad social y lo positivo que les ha dejado esta problemática al individuo y la comunidad.

De esta manera, se crean los espacios adecuados para la catarsis de lo que ha estado sucediendo, hacer diagnósticos de otras problemáticas, plantear posibles soluciones y dar una nueva mirada al medio ambiente. Estos hechos deben ser expresados de forma compartida para posibilitar la expresión de sentimientos, percepciones y posibles soluciones.

La ciénaga es la principal fuente de sostenimiento para las familias en situación de vulnerabilidad de Ayapel Córdoba; como es un recurso que se acaba, por tanto, se hace necesario que los Trabajadores de la Plaza del Mercado reflexionen y empiecen a corregir, prácticas, actitudes, relaciones, etc. conllevando a la disminución de la contaminación, y así también, a la conservación del recurso hídrico.

Tal vez no sea fácil reducir ahora la contaminación existente, tanto del agua, como del suelo, el aire, etc., pero si es más fácil que uno a uno se acostumbren a cuidar el agua, a reciclar, a repensar su actitud, su significado por lo que considera suyo, a valorar los recursos. Y a lo mejor en un mañana, no muy lejano, se respire un aire limpio en la Ciénaga, en la plaza de Mercado y porque no en todo el municipio de Ayapel.

En este caso se evidenció la falta de control del Estado ante el deterioro permanente dela ciénaga. Pero también debe existir el control social, donde la comunidad debe comenzar a exigir el cuidado del entorno, porque al fin $\mathrm{y}$ al cabo todos son habitantes de este municipio y de este gran planeta azul, que llamamos hogar. Hogar, que si no se hace algo, terminará por destruirse. Porque "Cuando el último árbol haya sido abatido, cuando el último río haya sido envenenado, cuando el último pez haya sido pescado, sólo entonces nos daremos cuenta de que no se puede comer el dinero" (Jefe Seattle, 1856). 


\section{Referencias}

1. COLOMBIA. AYAPEL-CÓRDOBA. Diagnóstico Técnico. En: Plan de Desarrollo Municipal, 2004-2007. 43-50, 89, 92, 157, 184, 197, 199, 212, 216, 235p.

2. Ibíd. p.43-50, 157.

3. BOYLE, J. Asuntos éticos en la etnografía en: MORSE, J. Asuntos críticos en los métodos de investigación cualitativa. Editorial Universidad de Antioquia. 2003 P.389-413.

4. COLOMBIA. AYAPEL-CÓRDOBA. Diagnóstico Técnico. En: Plan de Desarrollo Municipal, 2004-2007. 89p.

5. DESCHAMPS, J. C., MORALES, J. F., PAEZ, D. $y$ WORCHEL, S. identité sociale. La constrution de l'individu dans les relations entre groupes. Saint-Martin d'Héres, PUG. 1999. P.99.

6. OAKES, P. S., HASLAM, A. y TURNER, J. C. Construction de l'identité à partir du contexte. En: DESCHAMPS, J. C., MORALES, J. F., PAEZ, D. y WORCHEL, S. identité sociale. La constrution de l'individu dans les relations entre groupes. Saint-Martin d'Héres, PUG. 1999. P.111.

7. AGUDO GUEVARA, Álvaro. Nostalgia de la premodernidad. Historia de San Juan de las Galdonas en la memoria de habitantes mayores de 60 años. Ponencia presentada en la Segunda Reunión del Grupo Internacional de investigación sobre Representaciones Sociales e Imaginarios Latinoamericanos de la Maison des Sciences de I'Home, Río de Janeiro, del 31 de agosto al 01 de septiembre, 2003.

8. MEAD, George H. Mind, Self and Society. University of Chicago Press (editado por C. W Morris), Chicago, 1938. P.218.

9. BANCH, María Auxiliadora. Las representaciones sociales como perspectiva teórica para el estudio etnográfico de comunidades. En: VALENCIA ABUNDIZ, Silvia. Representaciones sociales: alteridad, epistemología y movimientos sociales. Primera edición. Universidad de Guadalajara, Maison de Sciences de L'Home Editions. ISBN 970-27-1005-7. 2006. P.217.

10. MEAD, George H. Mind, Self and Society. University of Chicago Press (editado por C. W Morris), Chicago, 1938. P.218.

11. JODELET D. La representación social: fenómenos, concepto y teoría. En: Psicología Social II. Barcelona: Editorial Paidós, 1988. p.75

12. WAGNER, W., DUVEEN, G., VERNA, J. y THEMEL, T. "I Have Some Fait and the Same Time I don't Believe. Cognitive
Poliphasia and Cultural Change". En: JCASP Special Issue: Health, Community and Development, 2000 p.4-5

13. FERDINAND VON HELMHOLTZ, Hermann Ludwig. Citado por: RELLA, Franco. Metamorfosis: Imágenes del pensamiento. Traducción de Joaquín Jordá. Madrid: Editorial Espasa-Calpe. 1989. p.46

14. URBINA, S. J. y FREGOSO, M. J. Afrontamiento de riesgos ambientales: El caso de San Juanico. Revista de Psicología Soc y Pers. 1991. p.7(1):46-59.

15. PUYA. Percepción social de los riesgos. Madrid: Fundación MAPFRE. 1995. 40p.

16. JODELET, Denise. Les répresentations sociales: phenomene, concept et theorie. En: MOSCOVICI, Serge. Psycologie Sociale. París, PUF. 1984. P.361

17. MOSCOVICI, Serge. Foreword. En:Herzlich, C., Health and Illness: a Social Psychological Analysis. Academic Press. Londres. 1973. P.xi.

18. COLOMBIA. MINISTERIO DEL MEDIO AMBIENTE OFICINA DE PARTICIPACIÓN COMUNITARIA, EDUCACIÓN AMBIENTAL Y POBLACIÓN. Yo Participo, Tú Participas, Todos Somos Parte, ¡Hagamos el Ambiente!: Lineamientos para una Política para la Participación Ciudadana en la Gestión Ambiental. Santafé de Bogotá Popayán, Julio de 1998. P.26.

19. SÁNCHEZ, Homero, Andrade Victoria. Educación Ambiental Ecología. México: Editorial Trillas. 1997. p.1-4.

20. COLOMBIA. AYAPEL-CÓRDOBA. Diagnóstico Técnico. En: Plan de Desarrollo Municipal, 2004-2007. 92p.

21. SILVANA DE ROSA, Annamaría. “Por quées importante?” notas inspiradas en una mirada reflexiva a la teoría de las representaciones sociales. En: VALENCIA ABUNDIZ, Silvia. Representaciones sociales: alteridad, epistemología y movimientos sociales. Primera edición. Universidad de Guadalajara, Maison de Sciences de L'Home Editions. ISBN 970-27-1005-7. 2006. P.114

22. Ibíd. p.412

23. VIAUD, Jean. Contribution a I'actualisation de la notion de Mémoire Colective. En: Stéphane, Laurence y Nicolas Roussiau (dirs.), La Mémoire Sociale. Identités et Représentations Sociales. Rennes. Presses Universitaires de Rennes. 2002. P.29

24. JIMÉNEZ HERRERO, Luís. En su obra: Desarrollo sostenible y economía ecológica. (1996). 


\section{Otra bibliografía consultada}

25. ARRUDA, Ángela. Movimientos sociales, síntomas $y$ protagonistas dela democracia. En: VALENCIA ABUNDIZ, Silvia. Representaciones sociales: alteridad, epistemología y movimientos sociales. Primera edición. Universidad de Guadalajara, Maison de Sciences de L'Home Editions. ISBN 970-27-1005-7. 2006. P.193.

26. COLOMBIA. BANCO DE LA REPÚBLICA. Notas Económicas Regionales Costa Caribe. Es una publicación de los Centros Regionales de Estudios Económicos del Banco de la República. Su propósito es mostrar la evolución de las principales actividades económicas de las regiones en Colombia. Centro Regional de Estudios Económicos - CREE. Número 03. Cartagena. Noviembre de 2004. 4p.

27. MOSCOVICI, Serge. El fenómeno de las representaciones sociales. En: Farr, R., Mocovici. S. European Studies in Social Psychology. París, Francia: Cambridge. University Press. 1984. $17 p$.
28. MOSCOVICI, Serge. La psychanalyse, son image et son public. París. PUF. 1976. (Primera Edición: 1961).

29. MOSCOVICI, Serge. Social Representations. Explorations in Social Psychology. Cambridge, Polity Press. 2000 P. 212-213.

30. RATNER, Carl. Activity as a Key Concept for Cultural Psychology. En: Culture \& Psychology. N². 1996 p.409, 412. Consultado en Internet en la dirección http://www.humboldt1. com/ cr2/jaan.htm.10/05/04

31. WAGNER, Wolfgang. Queries about social representations and constructions. En: Journal for the Theory of Social Behaviour. Número 26. Vol.2. 1996. P.108, 111.

Revista. Humanismo.Soc. 2013; 25: 22-36. 